\title{
Enhancing cognitive functioning in the elderly: multicomponent vs resistance training
}

\author{
Roberta Forte ${ }^{1,2}$ \\ Colin AG Boreham' \\ Joao Costa Leite ${ }^{3}$ \\ Giuseppe De Vito' \\ Lorraine Brennan ${ }^{3}$ \\ Eileen R Gibney ${ }^{3}$ \\ Caterina Pesce ${ }^{2}$
}

'Institute for Sport and Health, University College Dublin, Dublin, Ireland; ${ }^{2}$ Department of Human Movement and Sport Science, University of Rome "Foro Italico," Rome, Italy; ${ }^{3}$ Institute of Food and Health, University College Dublin, Dublin, Ireland
Correspondence: Caterina Pesce University of Rome "Foro Italico," Piazza L De Bosis I5, 00I35

Rome, Italy

Tel +390636733366

Fax +390636733362

Email caterina.pesce@uniroma4.it
This article was published in the following Dove Press journal:

Clinical Interventions in Aging

9 January 2013

Number of times this article has been viewed

Purpose: The primary purpose of this study was to compare the effects of two different exercise training programs on executive cognitive functions and functional mobility in older adults. A secondary purpose was to explore the potential mediators of training effects on executive function and functional mobility with particular reference to physical fitness gains.

Methods: A sample of 42 healthy community dwelling adults aged 65 to 75 years participated twice weekly for 3 months in either: (1) multicomponent training, prioritizing neuromuscular coordination, balance, agility, and cognitive executive control; or (2) progressive resistance training for strength conditioning. Participants were tested at baseline $\left(\mathrm{T}_{1}\right)$, following a 4-week control period $\left(\mathrm{T}_{2}\right)$, and finally at postintervention $\left(\mathrm{T}_{3}\right)$ for executive function (inhibition and cognitive flexibility) and functional mobility (maximal walking speed with and without additional task requirements). Cardiorespiratory and muscular fitness were also assessed as potential mediators.

Results: Indices of inhibition, the functions involved in the deliberate withholding of prepotent or automatic responses, and measures of functional mobility improved after the intervention, independent of training type. Mediation analysis suggested that different mechanisms underlie the effects of multicomponent and progressive resistance training. While multicomponent training seemed to directly affect inhibitory capacity, resistance training seemed to affect it indirectly through gains in muscular strength. Physical fitness and executive function variables did not mediate functional mobility changes.

Conclusion: These results confirm that physical training benefits executive function and suggest that different training types might lead to such benefits through different pathways. Both types of training also promoted functional mobility in older adulthood; however, neither inhibitory capacity, nor muscular strength gains seemed to explain functional mobility outcomes.

Keywords: executive function, functional mobility, physical training, physical fitness, aging

\section{Introduction}

Recently, considerable research has been directed at understanding the effects of physical exercise training on cognitive function and brain plasticity in older individuals. ${ }^{1}$ Findings have supported the view that executive cognitive functions, which are relevant for the control of goal-oriented actions and adaptive behaviors, are strongly impaired by aging, but also positively and specifically sensitive to aerobic exercise training. 2,3 This evidence has led to the formulation of the "selective improvement hypothesis," linking aerobic physical activity to specific executive function outcomes. However, the current opinion remains divided as to the causal link between gains in aerobic fitness and of the selective cognitive benefits. ${ }^{4-6}$ In fact, the search for potential mediators 
of chronic exercise effects on cognition is still ongoing with evidence emerging on the benefits of resistance training. ${ }^{\text {? }}$

Physical training studies targeted to promote cognitive functioning have been mainly based on varying the quantitative parameters of exercise (intensity, duration, and frequency) to identify a dose-response relationship between physical activity and cognitive performance. ${ }^{8}$ In contrast, not much attention has been paid to evaluate the nonphysical components of exercise (ie, the complexity of the cognitive or coordinative demands inherent in movement tasks), ${ }^{9}$ which might contribute to its cognitive outcomes. ${ }^{2,10}$ The paucity of longitudinal exercise interventions challenging neuromuscular coordination is surprising, since studies on motor training clearly demonstrate that movement task complexity and the related involvement of executive function have a strong impact on neuroplasticity and therefore on cognitive function. ${ }^{11}$ Indeed some evidence exists that cognitive benefits may be obtained by means of training programs that are not primarily focused on endurance and resistance, but on neuromuscular coordination and balance. ${ }^{12}$

Thus, the aim of the present study was to compare, in healthy community dwelling older individuals, the effects of two physical exercise interventions: one prioritizing the development of muscular resistance (progressive resistance training, PRT), and the other focusing on neuromuscular coordination, balance, agility, and cognitive executive control (multicomponent training, MCT). It was hypothesized that both exercise interventions would have a positive impact on executive functions and functional mobility. Furthermore, it was expected that the MCT would lead to more pronounced enhancements on executive functions and functional mobility due to greater and more variable coordinative requirements of the movement tasks leading to higher cognitive effort. ${ }^{12}$ In particular, attention was paid to the effects on inhibitory control, which represents one of the core executive functions. Specifically, among the wide variety of functions for which the term inhibition is commonly used, we refer to one's ability to deliberately inhibit dominant, automatic, or prepotent responses when necessary. Such ability plays a key role in instrumental daily life activity and social behavior control in elderly. ${ }^{13,14}$ Moreover, to explore the mechanisms underlying potential training effects, mediation hypotheses were examined. Firstly, it was tested whether or not traininginduced physical fitness changes mediated training effects on executive function. While the mediating role of aerobic fitness gain is still an issue of debate, ${ }^{4}$ the role of muscular strength gains has still not been investigated. Secondly it was tested whether or not gains in executive function and/or physical fitness translated into better functional mobility. A positive mediation was hypothesized given the role of both physical fitness and cognitive efficiency in determining functional mobility in older adults. ${ }^{15,16}$

\section{Methods}

\section{Participants}

Following approval from the local university ethics committee, community-dwelling individuals of both genders aged between 65 and 75 years were recruited. Different sources were used to avoid threat to generalizability due to homogeneous cultural level, namely local university alumni and parish newsletters, general practitioners' offices, and a consumer marketing database. The following inclusion criteria were applied: (1) not taking part in any regular physical exercise more than once a week; (2) being "medically stable" established via a medical history questionnaire, ${ }^{17}$ and (3) requiring the absence of uncontrolled cardiac illness, history of cerebrovascular disease, severe lower limb arthritis, uncontrolled metabolic disease, and other pathological conditions potentially influencing study outcomes. Moreover, potential participants were screened for daily living independence ability using the Lawton and Brody ${ }^{18}$ questionnaire. All included participants were fully functional, as was reflected by maximal scores in the above questionnaire. ${ }^{18}$

Of the 77 subjects who achieved these inclusion criteria, 50 started the program and eight either dropped out or were lost at follow-up (four belonging to the PRT and 4 to the MCT, with two participants lost for each reason in each training group). Reasons for discontinuing the intervention included the development of knee pain (one), anticipation of a scheduled operation (one), partner sickness (one), and no explanation (one). Two PRT participants and one MCT participant, although completing the allocated intervention, were lost at follow-up due to sickness on the day of testing. A further MCT participant had to be excluded for low compliance. Intent to treat analysis was not performed because there were no cases of false inclusion or of failure to start the intervention after random assignment, and because the number of individuals (eight) excluded from analysis after dropping out or lost at follow-up was balanced in the PRT and MCT groups.

Thus, 42 men and women (22 for MCT and 20 in PRT; mean age $69.8 \pm 3.4$ years; body mass index, $25.9 \pm 3.3 \mathrm{~kg} / \mathrm{m}^{2}$ ) comprised the actual sample (Table 1). Participants acted as their own controls, and thus no control group was recruited. After written informed consent was obtained, participants were randomly assigned to either MCT or PRT matching for 
Table I Main group characteristics and medical condition distributions across groups

\begin{tabular}{|c|c|c|}
\hline Group & $\begin{array}{l}\text { MCT } \\
n=22 ; F=15\end{array}$ & $\begin{array}{l}\text { PRT } \\
n=20 ; F=I I\end{array}$ \\
\hline \multirow[t]{2}{*}{ Age (years) } & $F=69.0 \pm 2.8$ & $F=70.5 \pm 3.9$ \\
\hline & $M=7 I .4 \pm 2.9$ & $M=69.1 \pm 3.7$ \\
\hline \multirow[t]{2}{*}{ BMI $\left(\mathrm{kg} / \mathrm{m}^{2}\right)$} & $F=26.2 \pm 3.1$ & $F=24.0 \pm 3.6$ \\
\hline & $M=27.1 \pm 2.9$ & $M=26.8 \pm 3.2$ \\
\hline \multirow[t]{2}{*}{ Stature $(\mathrm{cm})$} & $F=164.3 \pm 7.0$ & $F=162.6 \pm 6.1$ \\
\hline & $M=173.8 \pm 5.2$ & $M=172.8 \pm 5.5$ \\
\hline \multirow[t]{2}{*}{ Weight (kg) } & $F=70.6 \pm 6.2$ & $\mathrm{~F}=63.2 \pm 8.0$ \\
\hline & $M=81.9 \pm 10.1$ & $M=80.2 \pm 12.1$ \\
\hline \multirow[t]{3}{*}{ Pathologies } & Hypertension (5) & Hypertension (5) \\
\hline & High cholesterol (4) & High cholesterol (4) \\
\hline & Anemia (I) & Anemia (I) \\
\hline Chronic & Aspirin (5) & Aspirin (I) \\
\hline pharmacological & Statin (5) & Statin (2) \\
\hline \multirow[t]{4}{*}{ treatment } & ACE (2) & ACE (3) \\
\hline & Calcium antagonist (2) & Diuretics (2) \\
\hline & Diuretics (I) & Calcium antagonist (2) \\
\hline & & Bisphosphonates (I) \\
\hline Falls in previous & 4 subjects & 3 subjects \\
\hline \multicolumn{3}{|l|}{12 months* } \\
\hline Multiple & 4 (two drugs for & 3 (two drugs for \\
\hline treatment & hypertension or as & hypertension or \\
\hline & preventive; ie, aspirin & as preventive; ie, \\
\hline
\end{tabular}

Notes: The number of subjects is reported in parentheses. *No more than one fall was reported.

Abbreviations: $\mathrm{MCT}$, multicomponent training; PRT, progressive resistance training; n, number; $F$, female; $M$, male; BMI, body mass index; $A C E$, angiotensinconverting enzyme inhibitor.

gender and age. Following familiarization with test protocols, participants were assessed three times: at baseline $\left(\mathrm{T}_{1}\right)$, following a 4-week control period $\left(\mathrm{T}_{2}\right)$, and after a 12-week intervention $\left(\mathrm{T}_{3}\right)$ for the assessment of executive function, cardiorespiratory and muscular fitness, and walking speed under different conditions. For the total 16-week period of the study, participants were asked not to change their lifestyle, in particular regarding physical activity and diet.

\section{Measurements}

\section{Executive function assessment}

\section{Random number generation task}

The random number generation task evaluates executive functions, ${ }^{19}$ and particularly inhibition. The standard protocol for older individuals was adopted. ${ }^{20}$ Briefly, participants verbally generated a random sequence of 100 numbers chosen between one and nine at a frequency of $40 \mathrm{bpm}$ paced by a metronome. The randomness of the sequence was elaborated to obtain three indices that were theoretically related to the inhibitory function: the Turning Point Index, the Adjacency, and the Runs. ${ }^{21}$ The Turning Point
Index measures the similarity between the real frequency of turning points (change between ascending and descending series of numbers), and their theoretical frequency in random responses. The Adjacency measures the frequency of pairs of adjacent ascending or descending numbers, reflecting the habitual tendency to count forward or backward. The Runs is an index of variability of the number of digits in successive ascending or descending sequences. High levels on the Turning Point Index, but low levels of Adjacency and Runs correspond to a high ability to inhibit the production of stereotyped strings, therefore contributing to the optimal control of complex activities.

\section{Trail making test}

The trail making test assesses attention, speed, and cognitive flexibility. ${ }^{22}$ The standard protocol used requires participants to draw lines connecting in ascending order and as quickly as possible 25 circles distributed over a sheet of paper. ${ }^{22}$ The test has a part A with numbers only and a part B with numbers and letters joined in alternation (ie, 1-A-2-B-3-C, and so on). A summary score was calculated by subtracting the time taken in seconds to complete part A from the time at part B ( $\Delta$ Trail Making) and used for analysis.

\section{Cardiorespiratory fitness measurement}

Maximal oxygen uptake $\left(\mathrm{VO}_{2 \max }\right)$ was predicted using oxygen uptake $\left(\mathrm{VO}_{2}\right)$, and heart rate (HR) data obtained from an incremental cycling test up to $85 \%$ of the predicted maximal HR $\left(\mathrm{HR}_{\max }=220-\right.$ age $)$. During the test, participants breathed through a mask connected to a gas analyzer (Quark B2 COSMED, COSMED srl, Rome, Italy) to measure $\mathrm{VO}_{2}$, carbon dioxide, ventilation, and $\mathrm{HR}$. According to a standard protocol, following 2 minutes of measurement at rest, participants pedaled between 60 and $70 \mathrm{rpm}$ at 30 watts for 2 minutes, and then at workloads increased each minute by 10 and 15 watts for women and men, respectively. ${ }^{23}$ A 4-week test-retest reliability of predicted $\mathrm{VO}_{2 \max }$ was calculated as an intraclass correlation coefficient (ICC), obtaining a value of 0.88 .

\section{Muscular strength measurement}

Isokinetic maximal knee extension and flexion torques were measured on the dominant limb during four consecutive contractions at $60^{\circ} \mathrm{s}^{-1}$ (Biodex System Pro, Biodex Medical Systems, Inc, Shirley, NY, USA) following standard protocols..$^{24}$ Gravity correction was performed prior to each test, and verbal encouragement was given throughout the test. Maximal values were used for analysis. The 4-week 
test-retest ICC was 0.95 and 0.97 for knee extensor and flexor torques, respectively.

\section{Walking speed measurement}

Following the standard protocols reported in the literature for the assessment of maximal walking speed (MaxWS), ${ }^{25}$ the subject walked 10 meters on an indoor track "as fast as possible without running." The MaxWS was additionally measured under two different conditions adding an attentional demand: (1) talking (TalkWS), and (2) carrying an empty cardboard box (BoxWS). In the TalkWS task, participants were asked to name as many animals as they could think of beginning with either the letter B or C. This dual task assessment is commonly used in aging research. It evaluates the ability of older people to maintain efficient locomotion while they need to allocate capacity-limited cognitive resources to a concomitant mental task. All measurements were repeated twice, except for the TalkWS, for which a learning effect could be expected in the secondary task of naming animals. This could have led to a reduction in the cognitive load and a consequent alteration of the testing conditions. The best results were used for statistical analysis. ICC computations confirmed the stability over time (MaxWS, 0.85; BoxWS, 0.85 ; TalkWS, 0.64) generally reported in the literature. ${ }^{25}$

\section{Exercise program}

All participants exercised for 1 hour, twice weekly for 3 months. The MCT classes were comprised of a 15-minute warm up, leading to a 30 minute conditioning period of coordination/balance/strengthening/agility, followed by 30 minutes of floor exercises including stretching, strengthening, and relaxation. The classes aimed to stimulate overall function concurrently with perceptive and coordinative components of movement, combining elements of general mobility and coordination of the lower and upper limbs. Exercise was designed to ensure as much variety as possible since current prescription guidelines recommend the practice of multiple exercise regimes. ${ }^{26}$ Moreover, specific cognitive challenges were integrated into the physical training to engage executive function, and to specifically stimulate the inhibition of habitual responses and cognitive flexibility. For instance, participants had to perform task sequences while reversing or "scattering" a learned order, or to learn different stimulus-response associations and then switch between them according to external cues. Finally, given the demonstrated importance of reproducing the different challenges encountered in daily life, ${ }^{27}$ the proposed exercises required moving through space using walking progressively at faster speeds to light skipping, jogging, or with longer steps, and while adding the use of the arms. Difficulty was increased, for example, by walking while avoiding (ie, turning around) obstacles such as steps, hoops, hurdles, or people; negotiating (ie, climbing over) steps or obstacles (such as hurdles); or reducing the base of support as in normal/tandem walking performed either on a line marked on the floor, on skipping ropes placed on the floor, or in lines of aerobic steps.

The PRT session consisted of an initial 10 minutes during which participants actively warmed up by performing, while walking, movements of flexion, extension, and rotation of the joints to be used in the conditioning session. Thereafter, at each exercise session, participants completed in a circuit, a total of 12 strength exercises, alternating muscle groups and machines with free weights and floor exercises. These included machine exercises for knee extension, knee flexion, lateral pull-down, chest press, and front rowing; free weights and floor exercises for biceps, triceps, and deltoids, the lower limbs (ie, squats, stepping), abdominals, and back extension. At the machines, three sets of eight repetitions at $60 \%$ of one repetition maximum (1RM) for the first 2 weeks, and $80 \%$ $1 \mathrm{RM}$ thereafter, or at $15-17$ of the rate of perceived exertion, were performed for the entire training period. To maintain constant training intensity after the initial 2 weeks, the 1RM test was repeated every 4 weeks. For the other exercises, the same volume (sets by repetitions) was applied using body weight initially, and then progressively overloading with external devices (ie, weights disks or dumbbells) with intensity controlled by requiring participants to exercise at 15-17 of the rate of perceived exertion.

\section{Statistical analysis}

Data were analyzed with Predictive Analytics Software version 18 (SPSS Inc, Chicago, IL, USA). A significance level of $P<0.05$ was used in all computations. First, to test the effects of the interventions, all the measured variables were submitted to two-way analyses of variance with training type (MCT versus PRT) and testing time $\left(\mathrm{T}_{1}\right.$ versus $\mathrm{T}_{2}$ versus $\mathrm{T}_{3}$ ), as between and within-subjects factors, respectively. In case of significant interactions, post hoc analysis through planned paired $t$-tests was performed with Bonferroni correction for multiple comparisons $(P=0.25$ for two comparisons; $\mathrm{T}_{1}$ versus $\mathrm{T}_{2}$ and $\mathrm{T}_{2}$ versus $\left.\mathrm{T}_{3}\right)$. The same analysis model was applied to physical fitness $\left(\mathrm{VO}_{2 \max }\right.$, knee torques).

Second, to test whether postintervention improvements in physical fitness could explain improvements in executive function in both training groups, a mediational model 
with three regression analyses was applied to the executive function variables showing post-training increments. ${ }^{28}$ In the relationship between an independent and a dependent variable, a mediator is a third variable representing the mechanism through which the independent variable influences the dependent variable. In the present study, the validation of the mediation model implies that the effects of training type (categorical independent variable, $\mathrm{MCT}=1, \mathrm{PRT}=2$ ) on post-training changes in each executive function variable (dependent variable), is "explained" by post-training changes in physical fitness (mediator). Therefore, the three regressions of the model assessed whether: (1) training type had a significant effect on postintervention improvements in executive function (independent variable on the dependent variable); (2) training type had a significant effect on postintervention improvements in physical fitness (independent variable on the mediator); and (3) the prediction of executive function improvement accrued by training type was altered and became nonsignificant after including the physical fitness variable (independent variable and mediator on the dependent variable).

A similar, second mediational model was applied to the functional mobility measures showing post-training increments to verify whether postintervention improvements in physical fitness and/or executive function could account for improvements in functional mobility in both training groups. Therefore, the three regressions of the model assessed whether: (1) training type had a significant effect on postintervention improvements in functional mobility (independent on the dependent); (2) training type had a significant effect on postintervention improvements in physical fitness and/or executive function (independent on the mediators); and (3) the prediction of functional mobility improvement accrued by training type was altered and nonsignificant after including the physical fitness or executive function variable (independent and mediator on the dependent). The mediation significance and effect size were verified with the Sobel test. ${ }^{28}$

\section{Results}

Participants' compliance with the programs was $85 \%$ (20 of 24 sessions) for MCT and $86 \%$ (21 of 24 sessions) for PRT. Regarding the executive functions variables, a main time effect in the intervention period was observed for all variables (Table 2). Post hoc tests revealed a significant improvement after the intervention period (from $\mathrm{T}_{2}$ to $\mathrm{T}_{3}$ ) for the Turning Point Index $(t=-2.6, P=0.012)$ and Runs $(t=2.5, P=0.016)$. The Adjacency index showed improvements after both time
Table 2 Executive function measures (mean $\pm S D$ ) at $T_{1}, T_{2}$, and $T_{3}$

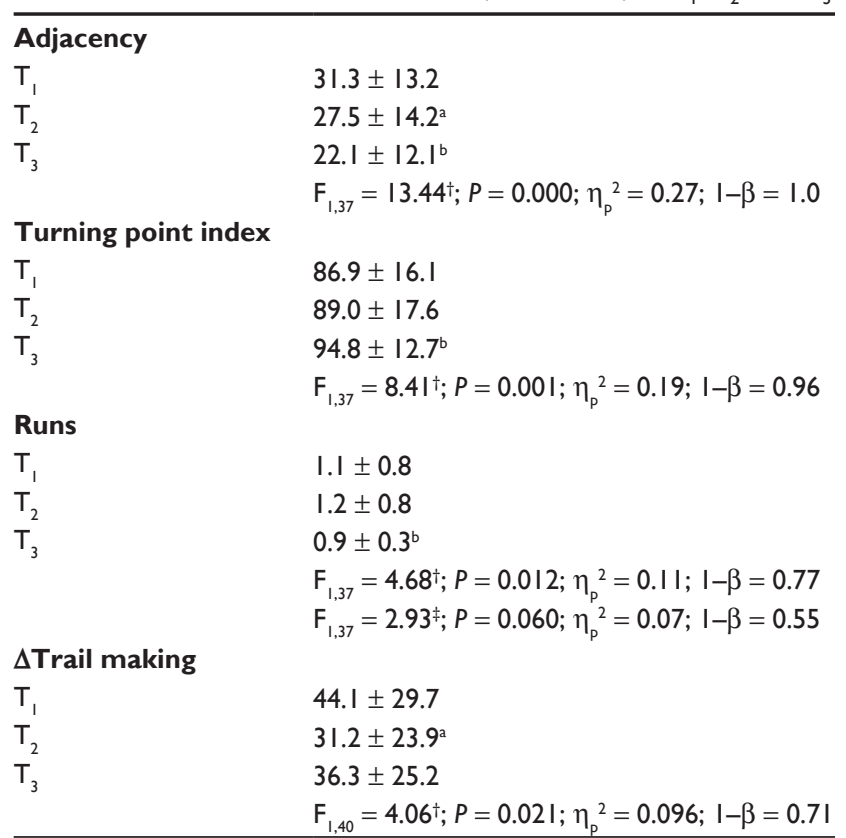

Notes: Analysis of variance and post hoc results are also reported. Data are collapsed across groups $(n=42)$; ${ }^{\dagger}$ main effect for time; ${ }^{\dagger}$ time*training interaction; ${ }^{a}$ significantly different from $T_{1}$; ${ }^{b}$ significantly different from $T_{2}$ at $P<0.025$ (Bonferroni correction).

Abbreviations: SD, standard deviation; $T_{1}$, baseline; $T_{2}$, control; $T_{3}$, following training: $\eta \mathrm{P} 2$, partial eta squared.

intervals $\left(\mathrm{T}_{1}\right.$ versus $\mathrm{T}_{2}, t=2.7, P=0.010 ; \mathrm{T}_{2}$ and $\mathrm{T}_{3}, t=2.7$, $P=0.011)$, whereas $\Delta$ Trail Making showed improvements between $\mathrm{T}_{1}$ and $\mathrm{T}_{2}$ only $(t=3.3, P=0.002)$. Moreover, for the Runs, the time by training interaction approached significance (Table 2). By visually inspecting the means separated by exercise group, a more marked improvement (ie, value reduction) could be observed for the PRT (-34\%) with respect to the MCT group (-8\%).

Regarding the functional mobility variables, a main time effect was observed for all, while no time by training interaction was found. Post hoc tests revealed improvements after the intervention period (from $\mathrm{T}_{2}$ to $\mathrm{T}_{3}$ ) for MaxWS $(t=-2.97, P=0.05)$ and BoxWS $(t=-2.87, P=0.006)$, while TalkWS did not change from $\mathrm{T}_{2}$ to $\mathrm{T}_{3}$, but changed from $T_{1}$ to $T_{2}$ (Table 3).

Regarding physical fitness, main time effects were observed for knee flexor and extensor torques, while a time by training interaction emerged for knee flexor torque only (Table 3). Post hoc analyses of the above interaction indicated a significant increment from $\mathrm{T}_{2}$ to $\mathrm{T}_{3}$ in the PRT group only $(t=3.46 ; P=0.001)$ (Table 3$)$.

Mediation analysis was run to determine whether postintervention improvements in physical fitness "explained" the effects of MCT and PRT on executive function. This mediation model with the three regressions was applied only to 
Table 3 Functional mobility measures (WS) and physical fitness (mean $\pm \mathrm{SD}$ ) at $\mathrm{T}_{1}, \mathrm{~T}_{2}$, and $\mathrm{T}_{3}$

\begin{tabular}{|c|c|}
\hline \multicolumn{2}{|c|}{ Maximal WS (m/second) } \\
\hline $\mathrm{T}_{1}$ & $1.91 \pm 0.21$ \\
\hline $\mathrm{T}_{2}$ & $1.95 \pm 0.24$ \\
\hline \multirow[t]{2}{*}{$\mathrm{T}_{3}$} & $2.03 \pm 0.22^{b}$ \\
\hline & $F_{1,40}=10.6^{\dagger} ; P=0.000 ; \eta_{p}^{2}=0.20 ; I-\beta=0.98$ \\
\hline \multicolumn{2}{|c|}{ Talking WS (m/second) } \\
\hline $\mathrm{T}_{1}$ & $1.65 \pm 0.24$ \\
\hline $\mathrm{T}_{2}$ & $1.74 \pm 0.26^{\mathrm{a}}$ \\
\hline \multirow[t]{2}{*}{$\mathrm{T}_{3}$} & $1.81 \pm 0.33$ \\
\hline & Box WS (m/second) \\
\hline $\mathrm{T}_{1}$ & $1.97 \pm 0.26$ \\
\hline $\mathrm{T}_{2}$ & $2.04 \pm 0.28$ \\
\hline \multirow[t]{2}{*}{$\mathrm{T}_{3}$} & $2.11 \pm 0.29^{b}$ \\
\hline & $F_{1,40}=9.7^{\dagger} ; P=0.000 ; \eta_{p}^{2}=2.00 ; I-\beta=0.98$ \\
\hline \multicolumn{2}{|c|}{$\mathbf{V O}_{2 \max }(\mathrm{mL} / \mathrm{kg} /$ minute $)$} \\
\hline $\mathrm{T}_{1}$ & $26.3 \pm 6.0$ \\
\hline $\mathrm{T}_{2}$ & $25.8 \pm 5.7$ \\
\hline \multirow[t]{2}{*}{$\mathrm{T}_{3}$} & $27.2 \pm 6.6$ \\
\hline & Knee extensors torque $(\mathrm{Nm})$ \\
\hline $\mathrm{T}_{1}$ & $100.2 \pm 30.8$ \\
\hline $\mathrm{T}_{2}$ & $101.3 \pm 32.0$ \\
\hline \multirow[t]{2}{*}{$\mathrm{T}_{3}$} & $107.6 \pm 20.1^{b}$ \\
\hline & Knee flexors torque $(\mathrm{Nm})$ \\
\hline $\mathrm{T}_{1}$ & $52.6 \pm 17.4$ \\
\hline $\mathrm{T}_{2}$ & $53.0 \pm 20.7$ \\
\hline \multirow[t]{2}{*}{$\mathrm{T}_{3}$} & $60.0 \pm 20.1^{b}$ \\
\hline & $\begin{array}{l}F_{1,40}=13.8^{\dagger} ; P=0.000 ; \eta_{p}^{2}=0.26 ; I-\beta=0.10 \\
F_{1,40}=6.5^{\ddagger} ; P=0.002 ; \eta_{p}^{2}=0.14 ; I-\beta=0.90\end{array}$ \\
\hline
\end{tabular}

Notes: Analysis of variance and post hoc results are also reported. Data are collapsed across groups $(n=42)$. †Main effect for time; ‘time*training interaction; ${ }^{a}$ significantly different from $T_{1}$; 'significantly different from $T_{2}$ at $P<0.025$ (Bonferroni correction).

Abbreviations: WS, walking speed; $S D$, standard deviation; $T_{1}$, baseline; $T_{2}$, control; $\mathrm{T}_{3}$, following training; $\eta \mathrm{p} 2$, partial eta squared; $\mathrm{VO}_{2 \max }$, maximal oxygen uptake.

those executive function variables, Turning Point Index and Runs, showing intervention effects ( $T_{2}$ to $T_{3}$ improvements) without potentially overlapping with test habituation/learning ( $\mathrm{T}_{1}$ to $\mathrm{T}_{2}$ improvements). The improvement in knee flexor torques was used as mediator as the only physical fitness variable showing a differential effect of MCT and PRT (ie, a time by training type interaction). Significant results of the mediation analysis are reported in Figure 1.

On the whole, these regression analyses showed that entering the gain in knee flexor torque into the model explained a significant part of the variance of the Turning Point Index and Runs scores, modifying the total effect of training type ( $\mathrm{R}^{2}$ change of the model for the Turning Point Index, 0.17-0.01 =0.16; for Runs, $0.35-0.09=0.26$; Figure 1). Specifically, these results indicate that the larger gains in knee flexor torque of the PRT group mediated the gains in the two inhibition indices. However, differences between the two mediation models applied to the Turning Point Index and Runs data concerned the type of mediation observed. For Runs (Figure 1B), a partial mediation emerged, as indicated by the reduction in the significance level of the direct training effects (unmediated effect coefficient $c$ versus mediated effect coefficient $\mathrm{c}_{1}$ ) when entering the mediator. In contrast, a different mediation pattern, referred to as inconsistent mediation, ${ }^{28}$ emerged for the Turning Point Index (Figure 1A). The total effect of training type on the Turning Point Index (c coefficient) was not significant because the direct effect ( $c_{1}$ coefficient) and the indirect mediated effect ( $a$ by $b$ coefficients) tended to cancel each other out. In fact, when subtracting from the total effect of training (c) the variance accounted for by the mediator, the remaining direct effect showed a coefficient $\left(c_{1}\right)$ of the sign opposite to that of the total effect (c). The direct effect of training on the Turning Point Index appeared to be in favor of the MCT group, while the mediated effect appeared to be in favor of the PRT group. This is indicated by the fact that, as MCT was set equal to 1 and PRT equal to 2, the sign of the total effect (c) was positive, and that of the remaining direct effect after applying mediation $\left(\mathrm{c}_{1}\right)$ was negative.

Finally, neither physical fitness nor executive functions mediated the effects of training type on functional mobility variables.

\section{Discussion}

The aim of the present study was to assess whether: (1) MCT and PRT interventions induced differential effects on executive functions and functional mobility in older adults; and (2) training effects on executive functions were mediated by physical fitness gains, and training effects on functional mobility were mediated by gains in physical fitness and/or executive functions.

On the whole, the results showed that both physical training types enhanced inhibitory capacity, that is, the deliberate, controlled suppression of prepotent responses and functional mobility. Furthermore, mediation analysis suggested the existence of both direct and indirect training effects on inhibitory functions, with MCT affecting them directly, and PRT effects being mediated by gains in muscular strength.

After the intervention period, both training groups exhibited significant improvements in all indices of inhibition. These improvements are attributable to the beneficial effects of the training intervention, since for two of three indices no improvements were observed between the two preintervention measurements. These findings support the growing 

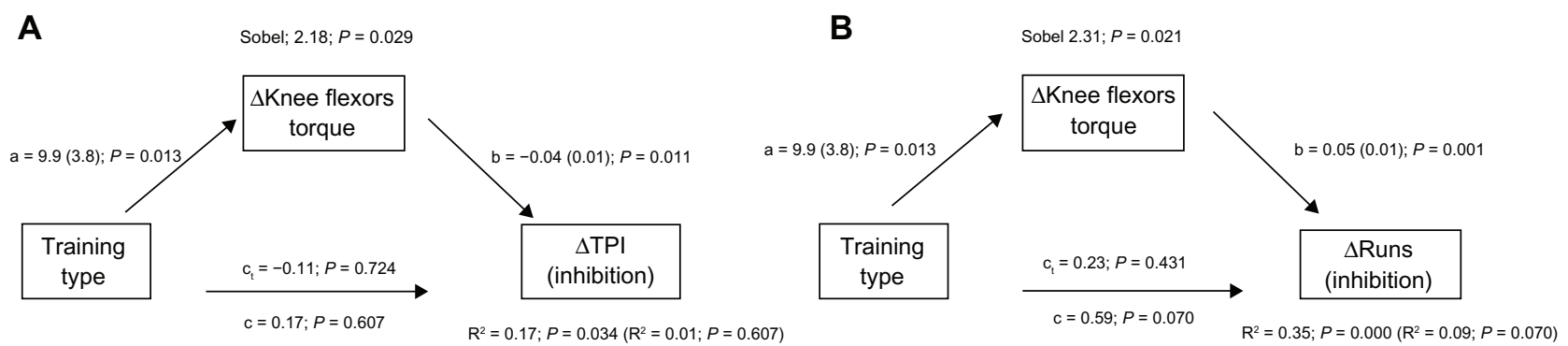

Figure I Mediation analysis for the effects of training type on posttraining gains in cognitive inhibitory function indexes mediated by posttraining gains in muscular strength. (A) TPI mediation model; (B) Runs mediation model.

Notes: Regression coefficients for changes in executive functions mediated by muscle strength gains (with SE in parentheses and associated $P$-values) are reported as follows: $a=$ effects of the independent variable on the mediator; $b=$ effect of the mediator on the dependent variable or total effect; $c=$ effect of the independent variable on the dependent variable; $c_{1}=$ the effect of the independent variable on the dependent variable after accounting for the mediator or the direct effect. $R^{2}$ values of regressions with the mediator (without the mediator in parentheses) and Sobel test results are also reported. $\Delta$ Knee flexors torque $=$ pre- and posttraining change in muscle strength. $\Delta \mathrm{TPI}=$ pre- and posttraining change in the TPI (inhibition index). $\Delta$ Runs = pre- and posttraining change in Runs (inhibition index).

Abbreviation: TPI, Turning Point Index.

evidence that physical exercise training benefits cognitive and particularly executive functions in aging (see Voss et al ${ }^{7}$ for a review).

The present findings also support the recently emerging indication that PRT positively influences executive functions, and particularly inhibitory capacity, ${ }^{7,29,30}$ challenging the traditional view that aerobic training is necessary to obtain cognitive benefits. ${ }^{4,5,31}$ Furthermore, while some mechanisms underlying aerobic fitness effects on executive functions have already been identified, ${ }^{8}$ and given that the exclusive role of aerobic fitness gains has been questioned, ${ }^{4}$ as of yet, no studies have searched for the mediators of resistance training effects on cognition. The present results suggest that gains in muscular strength may act as mediators of this relationship (Figure 1). It has to be considered, however, that the findings showed a partial mediation model, indicating that strength gains did not completely mediate the relationship between training type and inhibitory function. In fact, it can be speculated that additional factors may have contributed to the observed resistance training effects. For example, particularly for inexperienced individuals, resistance training implies information processing to recall exercise names, machine settings, principles of progression, and the muscle groups to be used, ${ }^{30}$ which may well represent a cognitive stimulus.

The mediation analysis also allowed for further differentiation between potential direct and indirect paths through which training benefited cognition, highlighting the specificity of the relationship between type of training, mediators, and cognitive outcomes. The key outcome of the present study emerged from the mediation pattern relative to the training effects on the Turning Point Index of inhibition (Figure 1A). The general effect of physical training on inhibitory function could be split into direct and indirect components: while PRT effects were mediated by gains in muscular strength, those of MCT appeared to be direct. This is in agreement with Davis et al, ${ }^{32}$ who argued that rather than being mediated by physical fitness benefits, the exerciseinduced cognitive changes may be a direct result of neural stimulation by cognitively challenging movements.

In contrast, regarding training effects on functional mobility, mediation analysis did not allow for the identification of any mediation pattern. Walking with or without concurring task demands (MaxWS) improved following training without the influence of either of the mediators under consideration.

The present study has limitations that should be addressed. First, a potential bias may derive from the fact that the study was not performed in a blinded fashion. Second, the results of the intervention effects might be limited by the relatively small sample size despite being estimated a priori using a power calculation. Regarding the mediation analysis, it is generally considered that it should be applied in large population studies; however, its application in small/medium sized research, such as the present study, is not unusual practice and does not necessarily hamper results. ${ }^{33}$ Third, volunteers were healthy and independent, characteristics which could have also reduced the impact of the interventions. Also, the observed increments from $T_{1}$ to $T_{2}$ in absence of $T_{2}$ to $T_{3}$ changes for the $\Delta$ Trail Making and TalkWS measures were probably due to a learning effect, rendering such measures inappropriate for studies in which participants act as their own controls. In the talking-while-walking task, a learning effect could be expected for the secondary task of naming animals, which may have generated a ceiling effect after the test was 
repeated twice. Moreover, it was unexpected that the Adjacency score, in contrast to the other two inhibition indices, significantly changed both from $\mathrm{T}_{1}$ to $\mathrm{T}_{2}$ and from $\mathrm{T}_{2}$ to $\mathrm{T}_{3}$; according to the literature, random number generation indices should be not influenced by practice effects, ${ }^{21}$ but this statement applies to the whole task and not to the individual indices. Finally, it may be possible that not only physical fitness mechanisms, but also other factors such as motivation, may mediate training effects on cognitive functions. In fact, motivated individuals might better allocate the available resources, allowing better task accomplishment. ${ }^{34}$

In conclusion, the applied forms of training seemed to have the common outcome of counteracting age-related decrements in cognitive executive functioning and performance on the selected functional mobility tasks. However, the paths leading to cognitive outcomes seemed to be different. It appeared that the beneficial effects of PRT on inhibition were mediated by gains in muscular strength, whereas the benefits of MCT were not. Speculatively, a direct cognitive stimulation by movement may be inherent in the motor tasks of the MCT, characterized by variable neuromuscular coordination and perceptual motor adaptations requirements. Moreover, cognitive control requirements had been generated through task sequence reversing/scattering and task switching demands.

Recently, coordination training has been demonstrated to positively affect executive functions, possibly through mechanisms different from those involved in cardiovascular training. ${ }^{10}$ Also, adding virtual reality cognitive challenges to stationary cycling has been proposed as a more efficacious mean than traditional physical exercise for improving executive functions in older adults. ${ }^{35}$

Combining physical and cognitive training components in sequence has been shown to maximize cognitive benefits in the elderly. ${ }^{36-38}$ Training programs integrating physical and cognitive components have also been tested, with the physical component consisting of stationary cycling or walking, ${ }^{39}$ or incorporating a more varied virtual dance imitation that involved the sensing of stimuli, paying attention, and making quick decisions. ${ }^{40}$ While one study was aimed at evaluating the cognitive outcomes, ${ }^{39}$ the other examined the effects on step execution. ${ }^{40}$ The present study examined both cognitive and functional mobility outcomes and potential mediators of beneficial effects of different training types. Future research should investigate what types of multifaceted exercises specifically promote executive function improvements and to what extent these translate into functional ability.

\section{Acknowledgments}

This work was supported by funding received from the Irish Research Council for Science, Engineering and Technology. The authors wish to thank Dr Maria Scatigna for her support and advice for statistical analysis. There are no professional relationships with companies or manufacturers who will benefit from the results of the present study.

\section{Disclosure}

The authors report no conflicts of interest in this work.

\section{References}

1. Chodzko-Zajko W, Kramer AF, Poon LW, editors. Enhancing Cognitive Functioning and Brain Plasticity. Champaign, IL: Human Kinetics; 2009.

2. Kramer AF, Hahn S, Cohen NJ, et al. Ageing, fitness and neurocognitive function. Nature. 1999;400(6743):418-419.

3. Hillman CH, Erickson KI, Kramer AF. Be smart, exercise your heart: exercise effects on brain and cognition. Nat Rev Neurosci. 2008;9(1):58-65.

4. Etnier JL, Nowell PM, Landers DM, Sibley BA. A meta-regression to examine the relationship between aerobic fitness and cognitive performance. Brain Res Rev. 2006;52(1):119-130.

5. Angevaren M, Aufdemkampe G, Verhaar HJ, Aleman A, Vanhees L. Physical activity and enhanced fitness to improve cognitive function in older people without known cognitive impairment. Cochrane Database Syst Rev. 2008;3:CD005381.

6. Smiley-Oyen AL, Lowry KA, Francois SJ, Kohut ML, Ekkekakis P. Exercise, fitness, and neurocognitive function in older adults: the "selective improvement" and "cardiovascular fitness" hypotheses. Ann Behav Med. 2008;36(3):280-291.

7. Voss MW, Nagamatsu LS, Lui-Ambrose T, Kramer AF. Exercise, brain, and cognition across the life span. J Appl Physiol. 2011;11(5): 1505-1513.

8. Etnier JL. Physical activity programming to promote cognitive function: are we ready for prescription? In: Chodzko-Zajko W, Kramer AF, Poon LW, editors. Enhancing Cognitive Functioning and Brain Plasticity. Champaign, IL: Human Kinetics; 2009: 159-176.

9. Pesce C. Shifting the focus from quantitative to qualitative exercise characteristics in exercise and cognition research. J Sport Exerc Psychol. 2012;34:766-786.

10. Yan JH, Zhou CL. Effects of motor practice on cognitive disorders in older adults. Eur Rev Aging Phys Act. 2009;6(2):67-74.

11. Carey JR, Bhatt E, Nagpal A. Neuroplasticity promoted by task complexity. Exerc Sport Sci Rev. 2005;33(1):24-31.

12. Voelcker-Rehage C, Godde B, Staudinger UM. Cardiovascular and coordination training differentially improve cognitive performance and neural processing in older adults. Front Hum Neurosci. 2011;17(5):26.

13. Vaughan L, Giovanello K. Executive function in daily life: Age-related influences of executive processes on instrumental activities of daily living. Psychol Aging. 2010;25(2):343-355.

14. Von Hippel W. Aging, executive functioning, and social control. Curr Dir Psychol Sci. 2007;16(5):240-244.

15. Bean JF, Leveille SG, Kiely DK, Bandinelli S, Guralnik JM, Ferrucci L. A comparison of leg power and leg strength within the InCHIANTI study: which influences mobility more? J Gerontol A Biol Sci Med Sci. 2003;58(8):728-733.

16. Ble A, Volpato S, Zuliani G, et al. Executive function correlates with walking speed in older persons: the InCHIANTI study. J Am Geriatr Soc. 2005;53(3):410-415. 
17. Greig CA, Young A, Skelton DA, Pippet E, Butler FM, Mahmud SM. Exercise studies with elderly volunteers. Age Ageing. 1994;23(3): $185-189$.

18. Lawton MP, Brody EM. Assessment of older people: self-maintaining and instrumental activities of daily living. Gerontologist. 1969;9(3): 179-186.

19. Baddeley A, Emslie H, Kolodny J, Duncan J. Random generation and the executive control of working memory. $Q J$ Exp Psychol $A$. 1998;51(4):819-852.

20. Albinet C, Tomporowski PD, Beasman K. Aging and concurrent task performance: cognitive demand and motor control. Educ Gerontol. 2006;32(9):689-706.

21. Towse JN, Valentine JD. Random generation of numbers: a search for underlying processes. Eur J Cogn Psychol. 1997;9(4):381-400.

22. Strauss E, Sherman EMS, Spreen O. Trail Making Test (TMT). In: Strauss E, Sherman EMS, Spreen O, editors. A Compendium of Neuropsychological Tests: Administration, Norms, and Commentary, 3rd ed. New York: Oxford University Press; 2006:655-677.

23. Lenti M, De Vito G, Sbriccoli P, Scotto di Palumbo A, Sacchetti M. Muscle fibre conduction velocity and cardiorespiratory response during incremental cycling exercise in young and older individuals with different training status. J Electromyogr Kinesiol. 2010;20(4):566-571.

24. Drouin JM, Valovich-mcLeod TC, Shultz SJ, Gansneder BM, Perrin DH. Reliability and validity of the Biodex system 3 pro isokinetic dynamometer velocity, torque and position measurements. Eur J Appl Physiol. 2004;91(1):22-29.

25. Shumway-Cook A, Guralnik JM, Phillips CL, et al. Age-associated declines in complex walking task performance: the Walking InCHIANTI toolkit. J Am Geriatr Soc. 2007;55(1):58-65.

26. Baker MK, Atlantis E, Fiatarone Singh MA. Multi-modal exercise programs for older adults. Age Ageing. 2007;36(4):375-381.

27. Shumway-Cook A, Patla AE, Stewart A, Ferrucci L, Ciol MA, Guralnik JM. Environmental demands associated with community mobility in older adults with and without mobility disabilities. Phys Ther. 2002;82(7):670-681.

28. MacKinnon DP, Fairchild AJ, Fritz MS. Mediation analysis. Annu Rev Psychol. 2007;58:593-614.

29. Liu-Ambrose T, Nagamatsu LS, Graf P, Beattie BL, Ashe MC, Handy TC. Resistance training and executive functions: a 12-month randomized controlled trial. Arch Intern Med. 2010;170(2):170-178.
30. Liu-Ambrose T, Nagamatsu LS, Voss MW, Khan KM, Handy TC Resistance training and functional plasticity of the aging brain: a 12-month randomized controlled trial. Neurobiol Aging. 2012;33(8): 1690-1698.

31. Smith PJ, Blumenthal JA, Hoffman BM, et al. Aerobic exercise and neurocognitive performance: a meta-analytic review of randomized controlled trials. Psychosom Med. 2010;72(3):239-252.

32. Davis CL, Tomporowski PD, McDowell JE, et al. Exercise improves executive function and achievement and alters brain activation in overweight children: a randomized, controlled trial. Health Psychol. 2001;30(1):91-98

33. Rucker DD, Preacher KJ, Tormala ZL, Petty RE. Mediation analysis in social psychology: current practices and new recommendations. Soc Personal Psychol Compass. 2011;5/6:359-371.

34. Humphreys MS, Revelle W. Personality, motivation, and performance: a theory of the relationship between individual differences and information processing. Psychol Rev. 1984;91(2):153-184.

35. Anderson-Hanley C, Arciero PJ, Brickman AM, et al. Exergaming and older adult cognition: a cluster randomized clinical trial. Am J Prev Med. 2012;42(2):109-119.

36. Fabre C, Massé-Biron J, Chamari K, Varray A, Mucci P, Préfaut C. Evaluation of quality of life in elderly healthy subjects after aerobic and/or mental training. Arch Gerontol Geriatr. 1999;28(1):9-22.

37. Fabre C, Chamari K, Mucci P, Massé-Biron J, Préfaut C. Improvement of cognitive function by mental and/or individualized aerobic training in healthy elderly subjects. Int J Sports Med. 2002;23(6):415-421.

38. Oswald W, Gunzelmann T, Rupprecht T, Hagen B. Differential effects of single versus combined cognitive and physical training with older adults: the SimA study in a 5-year perspective. Eur J Ageing. 2006; 3(4):179-192.

39. Schaefer S, Schumacher V. The interplay between cognitive and motor functioning in healthy older adults: findings from dual-task studies and suggestions for intervention. Gerontology. 2011;57(3):239-246.

40. Pichierri G, Coppe A, Lorenzetti S, Murer K, de Bruin ED. The effect of a cognitive-motor intervention on voluntary step execution under single and dual task conditions in older adults: a randomized controlled pilot study. Clin Interv Aging. 2012;7:175-184.
Clinical Interventions in Aging

\section{Publish your work in this journal}

Clinical Interventions in Aging is an international, peer-reviewed journal focusing on evidence-based reports on the value or lack thereof of treatments intended to prevent or delay the onset of maladaptive correlates of aging in human beings. This journal is indexed on PubMed Central, MedLine, the American Chemical Society's 'Chemical Abstracts

\section{Dovepress}

Service' (CAS), Scopus and the Elsevier Bibliographic databases. The manuscript management system is completely online and includes a very quick and fair peer-review system, which is all easy to use. Visit http://www.dovepress.com/testimonials.php to read real quotes from published authors. 